\title{
Expanding the FDS Simulation Capabilities to Fire Tunnel Scenarios Through a Novel Multi-scale Model
}

\author{
Vittorio Verda (10*, Dipartimento Energia, Politecnico di Torino, c.so Duca degli \\ Abruzzi 24, 10129 Torino, Italy \\ Romano Borchiellini, Dipartimento Energia, Politecnico di Torino, Torino, Italy \\ Sara Cosentino, Tecnositaf S.p.A., Torino, Italy \\ Elisa Guelpa and Jesus Mejias Tuni, Dipartimento Energia, Politecnico di \\ Torino, Torino, Italy
}

Received: 24 January 2020/Accepted: 15 December 2020/Published online: 3 May 2021

\begin{abstract}
Computational Fluid Dynamics (CFD) is widely used to simulate tunnels and partially substitute on-site tests. As technology advances, new application opportunities appear; some examples are the optimal operation of ventilation and emergency systems, risk assessment of tunnels and training of the operators. Even when the computational capacity of computers has grown, CFD is still constrained by the large amount of computational resources needed in long tunnels. This introduces a need for methods able to reduce the amount of time required for simulations. To face this need, a novel 1D-3D multiscale model is presented in this paper. The model incorporates the code Whitesmoke into FDS (Fire Dynamics Simulator) through a direct coupling. Whitesmoke manages the fluid dynamics, temperature and concentration of species in the 1D portion, while FDS calculates these fields in the portion where fire occurs. Using this multiscale model, the computation time for long tunnels is reduced, proportionally to the $1 \mathrm{D}$ length in the domain. Also, additional simulation capabilities particularly useful for tunnel analysis are obtained. Some new characteristics are pressure boundary conditions can be easily imposed at the tunnel portals or at the ventilation shafts; the characteristic curves of the fans/jet-fans can be included, also considering the degradation effects due to smoke propagation; the piston effect can be properly considered. Our research verifies most of its capabilities, also clarifying its limitations and the criteria used to set the domain for the analysis. As a final step, the model is tested in a tunnel with a cross section of $4.8 \mathrm{~m}$ and $600 \mathrm{~m}$ of length with a $2 \mathrm{MW}$ fire, comparing its performance with a full 3D FDS simulation. The difference in temperature and velocity is minimal for most of the domain, making It a good way to optimize resource usage in large simulations. Furthermore, the multiscale manages to reduce the computational time of more than a $50 \%$.
\end{abstract}

Keywords: Multiscale model, FDS, WhiteSmoke, Tunnel fire simulation

\footnotetext{
*Correspondence should be addressed to: Vittorio Verda, E-mail: vittorio.verda@polito.it
} 


\section{Introduction}

Computational Fluid Dynamics (CFD) software packages have been constantly used to simulate tunnels and substitute on-site tests. With the advancement of technology also new applications are developed like ventilation and emergency systems management in tunnels, risk assessment and VR training. Despite the continuously growing calculation speed of computers, CFD remains time costly, especially for long tunnels. Because of this, CFD calculations are still unable to give fast-enough results for these applications and need some developments that help shortening simulation times.

Simulation of fire scenarios of different characteristics has represented an intensely researched topic in the last years. The attempts started with 1D models like MFIRE [1], SPRINT [2], WHITESMOKE [3-5], which have been applied to underground network structures, such as tunnels or mines. Then, zone models have been developed, BRANZFIRE [6], FSSIM [7], CFAST [8, 9], focusing in simulating fires in compartments that might be linked in a network, like rooms in a building. Still, in several applications, the main approach to simulate fires in the actuality is CFD, as it tends to be more precise and describe the physics in a more accurate way.

CFD has been largely used to simulate fires from confined fires [10, 11], to extinction modelling [12] and tunnel fires. Regarding tunnel fires, most applications aim at providing practical evaluations of phenomena such as smoke movement in tilted tunnels, as in [13], and backlayering, as in [14, 15]. Another application field is the reconstruction of real fires, as in [16], where the Hsuehshan tunnel fire has been simulated using FDS. Still long tunnels represent an issue for CFD simulations, because large domains require large computational resources, while simulations might take days or weeks to complete. These issues can be lessened using multiscale modelling.

Multiscale modelling is understood as the group of strategies to model tunnels by combining $3 \mathrm{D}$ simulations in a portion of the tunnel and $1 \mathrm{D}$ simulations in the remaining part, to obtain a calculation time reduction. Although reduction in computational resources is generally considered as the main goal, there are additional advantages in using multiscale modeling, as it enhances the simulation capabilities with respect to full CFD models, as discussed later in this paper. Some research on multiscale methods has been conducted in the last decades. Some multiscale applications have aimed towards the calculation of pressure in linked pressure vessels using 3D and 1D calculations, as TRAC (Transient Reactor Analysis Code) [17], and GOTHIC [18]. This last one also used as a base to the FDS-HVAC solver [19]. Other applications of Multiscale modelling are found also in medical research as [20], where the circulatory system near the brain is simulated by using a 1D for the circle of Willis and a 3D for the carotid artery. The first applications, of multiscale models with fire and ventilation systems, were conducted at Politecnico di Torino combining the CFD software Fluent with 1D equations written as User Defined Functions. In [21] the multiscale method is presented, and the different coupling schemes are compared. The analysis is mainly 
focused on the thermal effects of the fire, which are further discussed also in [22]. In [3] and [23] the effects of ventilation are also discussed. Further developments have then tried to use the code Fire Dynamic Simulator (FDS), instead of Fluent. The main reasons are related with the fact that FDS is an open-source code and it is widely used in the community of fire engineering. In [24], FDS is coupled with the 1D code Vent Fire. Both direct and indirect coupling are tested, but an indirect approach is selected. This option is faster than the direct coupling but has the demerit of needing time to obtain the characteristics curves of the system, to divide it in an appropriate way. In [25], FDS is coupled with its own 1D tool, the HVAC, analyzing the time reduction in cold simulations. In [26], the HVAC tool is adopted with the goal of analyzing the reductions in the computational time that can be obtained through MPI parallel computation.

Still the results achieved using FDS in a multiscale framework are not at the same level as those obtained with Fluent. The main reasons are related with the limitations of the HVAC tool while performing direct coupling. The HVAC tool does not allow one to consider the temperature evolution in the 1D portion, thus impeding proper analysis of fans installed in the tunnel or ventilation channels where smoke propagates. In addition, the tool makes it difficult to consider pressure boundary conditions at the portals, which is the most appropriate condition, especially in operational analysis. The present article tackles these issues and presents a fully coupled Multiscale algorithm that incorporates the CFD capabilities of FDS and the 1D code Whitesmoke. Both computational tools are written in Fortran language, compiled together and integrated to achieve the maximum reduction in calculation time. Furthermore, Whitesmoke can calculate the 1D flow, including species transport, thermal losses in the tunnel, calculation of inclined tunnels and placement of fans and obstacles, adding capabilities to the Multiscale simulations. The application range of this novel tool includes several engineering fields. Particularly our aim is its use in tunnel fires and ventilation systems, to simulate this kind of structures in a more efficient way. These tunnels include vehicular tunnels, mining tunnels, metro tunnels and every other subterraneous and subaquatic tunnel.

\section{Modelling Approach}

\subsection{General Description of the Algorithm}

In this work, a multiscale model is created. This model is able to fully exploit the capabilities of both 3D and 1D modelling, in terms of accuracy in front of complex cases and reduced calculation time. The model integrates the 1D software Whitesmoke, which is directly introduced in FDS.

The algorithm works as shown in Fig. 1. FDS and the 1D model are ran in series, using the $3 \mathrm{D}$ with boundaries imposed by the $1 \mathrm{D}$, and inversely, running the 1D with 3D data as the boundaries. Both algorithms run successively every few seconds to achieve the coupling of their results. At first, the 1D model is run at $\mathrm{t}=0$ in order to obtain the boundary conditions to be considered in the $3 \mathrm{D}$ model, as discussed in the following sections. As the time-step adopted in the 1D 


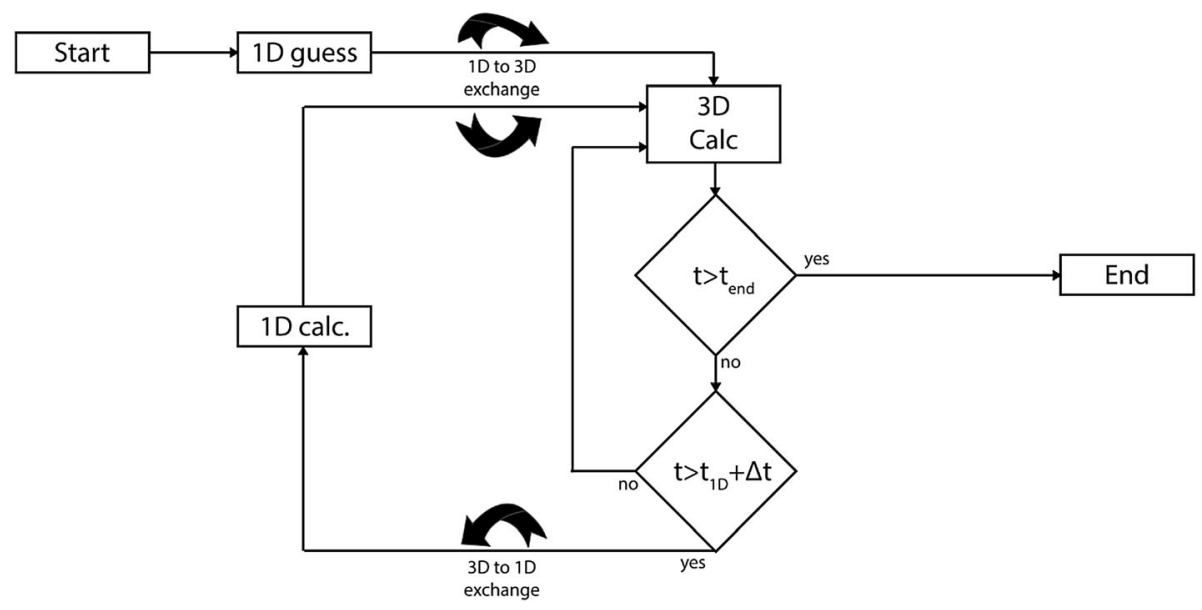

\section{Figure 1. Multiscale Algorithm Representation.}

model is larger than that used in the 3D model, the latter is run using fixed boundary conditions until the time-step of the 1D model is reached. After that, the $1 \mathrm{D}$ model is run using the boundary conditions obtained from the $3 \mathrm{D}$ model. The 1D model determines the new conditions for the 3D model. This procedure continues until the simulation is completed.

This time stepping structure allows for more stability, as a change in the boundary conditions introduces a change in the whole domain of the CFD simulation. Running both simulations together would require iterating more times the CFD part of the code, to achieve convergence, reducing the potential time reducing benefits of the whole algorithm.

\subsection{D descriptions and Characteristics}

Whitesmoke is responsible for the simulation of the far-field. This model purpose is to solve three groups of equations, referred to the fluid dynamic, thermodynamic and mass-transport in its 1D field. Each of these solution procedures uses specific approaches. In this section, two notations are used, first, for the NavierStokes and concentration equations, plain letters are scalars, bold letters are vectors and letters with and arrow on top are tensors. The second notation is the matrix notation, used for the network representation of the equations, here the variables include a sub-index that indicates their dimension and origin.

2.2.1. Network Structure This method can represent, in a compact way, systems where one dimension is preponderant over the other two dimensions [4]. The Graph theory [27] is used to describe the topology of these systems. The domain is defined using nodes and branches. Each portion of the tunnel or ventilation system is represented as a branch, bounded by two nodes (the inlet node and the outlet node). In the branches, the physical and geometrical properties are defined (length, diameter, rugosity) and the flow rates. The nodes represent the extremes 
of the branches and link the branches among them. In each node, the pressure, temperature and composition are defined. At last, a matrix can be built using these nodes and branches, called the incidence matrix. This matrix has one row for each node and one column for each branch. In each column, the inlet (with $\mathrm{a}+1)$ and outlet $(-1)$ nodes of each branch are identified. These are conventionally defined, meaning that the flow rate in a branch is positive if it flows from the inlet towards the outlet and negative otherwise.

2.2.2. Fluid Dynamics Model This part of the model is built around a modified version of the 3D time dependent Navier-Stokes equations for continuity (2.1) and momentum (2.2).

$$
\begin{aligned}
& \frac{\partial \rho}{\partial t}+\nabla \cdot(\rho \boldsymbol{u})=0 \\
& \frac{\partial \rho \boldsymbol{u}}{\partial t}+\nabla \cdot(\rho \boldsymbol{u} \boldsymbol{u})=-\nabla p+\nabla \cdot \vec{\tau}+\Delta P_{\text {source }}
\end{aligned}
$$

where $\rho$ is the density, $\mathbf{u}$ the velocity, $\mathrm{t}$ the time, $\mathrm{p}$ is the pressure and $\nabla . \vec{\tau}$ is the viscous term. Proper assumptions are considered for the Navier-Stokes equations; in particular, the one-dimensional form of these equations is considered, for continuity (2.3) and momentum (2.4). Two momentum sources are introduced $\Delta P_{\text {fric }}$. and $\Delta P_{\text {source }}$. The first one, the $\Delta P_{\text {fric }}$, in order to account for the viscous term which losses most of its meaning when eliminating the transversal dimensions, it accounts for local and distributed friction. The second is the $\Delta P_{\text {source }}$. and mainly accounts for momentum generated by fans, jetfans and similar sources.

$$
\begin{aligned}
& \frac{d \rho}{d t}+\frac{\mathrm{d} \rho \mathrm{u}}{\mathrm{dx}}=0 \\
& \rho \frac{d u}{d t}+\rho u \frac{d u}{d x}=-\frac{d p}{d x}-\Delta P_{\text {fric }}+\Delta P_{\text {source }}
\end{aligned}
$$

The equations are developed around branches (momentum) or nodes (continuity), following the network representation. The backward Euler method is used as the time advancing scheme. The final equations are Eq. (2.5), for continuity, and Eq. (2.6), for momentum.

$$
\frac{\left(\rho_{i}^{t}-\rho_{i}^{t-\Delta t}\right)}{\Delta t}\left(\sum_{j} \frac{A_{j} L_{j}}{2}\right)+\sum_{j} u_{j} A_{j} \rho_{j}+G_{e x t, i}=0
$$




$$
\begin{aligned}
& \rho_{j} \frac{\left(u_{j}^{t}-u_{j}^{t-\Delta t}\right)}{\Delta t} L_{j}+\left(P_{i+1}-P_{i}\right)-\Delta P_{\text {fric } j}+\Delta P_{\text {source } j}=0 \\
& P_{i}=p_{i}+\frac{\rho_{i} u_{i}^{2}}{2}+\rho_{i} g z_{i}
\end{aligned}
$$

where $\mathrm{L}$ is the length of the branch, A the cross section, $\mathrm{G}$ the mass flow rate, $\mathrm{i}$ enumerates the nodes and $\mathrm{j}$ the branches. Equation (2.7) shows the meaning of the total pressure, that includes the static pressure, the dynamic pressure and the buoyancy term.

Proper semi-empirical relations are used in order to compute the two source terms. In the case of the distributed and local losses due to friction, $\Delta P_{\text {fric }}$, Eq. (2.8) is used, from [28].

$$
\Delta P_{\text {fric } j}=\frac{1}{2}\left(f_{j} \frac{L_{j}}{D_{h, j}}+\sum \beta_{j}\right) \rho_{j} u_{j}^{2}
$$

In (2.8) $f_{j}$ stands for the friction coefficient of the branch $\mathrm{j}, \beta_{j}$ the minor losses coefficient and $D_{h, j}$ the hydraulic diameter of the branch.

The term $\Delta P_{f a n}$ is calculated using two formulas, Eqs. (2.9) and (2.10). In these formulas a, b, c and d represent empirical coefficients that describe the polynomial function of a jet-fan, $n_{j}$ the amount of fans in branch $\mathrm{j}, A_{f}$ the fan discharging area, $K_{f}$ the pressure rise coefficient and $u_{f}$ the fan discharge velocity.

$$
\begin{aligned}
\Delta P_{\text {fan } j} & =a u_{j}^{3}+b u_{j}^{2}+c u_{j}+d \\
\Delta P_{\text {fan } j} & =n_{j} \rho_{j} \frac{A_{f}}{A_{j}} K_{f} u_{f}\left(u_{f}-u_{j}\right)
\end{aligned}
$$

The term $\Delta P_{\text {piston }}$ is evaluated through the expression (2.11). In this expression $\varepsilon_{v}$ stands for the aerodynamic factor of a determined vehicle, $A_{v}$ for that vehicle cross-section, $N_{1}$ and $N_{2}$ are the number of vehicles in each direction, and $u_{1}$ and $u_{2}$ the velocities in each corresponding direction. This calculation is solved for each kind of vehicle in the tunnel. This expression is extracted from [29].

$$
\Delta P_{\text {piston } j}=\varepsilon_{v} \frac{A_{v}}{A_{j}} \frac{\rho_{j}}{2}\left[N_{1}\left(u_{1}-u_{j}\right)^{2}-N_{2}\left(u_{2}-u_{j}\right)^{2}\right]
$$

The pressure variation terms used in the calculation are updated in each $1 \mathrm{D}$ timestep, updating the density and velocity values, introducing the effect of the degraded local conditions through the simulation. More detailed explanations of the developments shown in this part can be found in $[4,30]$. 
2.2.3. Thermal Model The thermal model is based on the transient energy Eq. (2.12).

$$
\frac{\partial\left(\rho c_{p} T\right)}{\partial t}+\nabla \cdot\left(\rho c_{p} \boldsymbol{u} T\right)=\nabla \cdot(k \nabla T)+\varphi_{v}
$$

where $\mathrm{c}_{\mathrm{p}}$ is the specific heat, $\mathrm{T}$ the temperature, $\mathrm{k}$ the thermal conductivity and $\varphi_{v}$ the volumetric source term. The development of the equation follows similar steps as the development of the fluid dynamic model. First, the equation is written in one-dimensional form (2.13):

$$
\rho c_{p} \frac{d T}{d t}+\rho c_{p} u \frac{d T}{d x}=k \frac{d^{2} T}{d x^{2}}+\varphi_{v}-\varphi_{l}
$$

The last term considers the heat losses through the walls, that cannot be explicitly calculated due to the absence of thermal gradients in transversal direction. Equation (2.13) is then integrated and discretized in the control volumes, defined as the volumes surrounding each node of the network. Developing the first term on the left-hand side using the Backward Euler method, drives to Eq. (2.14):

$$
\rho_{i}^{t} c_{p, i} \frac{\left(T_{i}^{t}-T_{i}^{t-\Delta t}\right)}{\Delta t} \Delta V+\sum_{j} \rho_{j}^{t} c_{p, i} u_{j}^{t} A_{j} T_{j}^{t}=\left.\sum_{j} k_{j} \frac{\partial T^{t}}{\partial x}\right|_{j} A_{j}+\phi_{v, i}-\phi_{l, i}
$$

It must be noted that the second term on the left-hand side of Eq. (2.14) accounts for the flows of energy entering and leaving the volume. Temperature in the cross section in the middle of each branch is evaluated using an upwind scheme, consequently it is equal to the temperature at the inlet node of the branch. The development of the equations is explained in deep in $[3,4,30]$.

The procedure followed to calculate the heat losses through the walls uses a global heat transfer coefficient to calculate the heat loss through the surface, as seen in Eq. (2.15) where $\Omega_{j}$ is the perimeter of the branch and $U_{j}$ the global heat transfer coefficient of the branch.

$$
\phi_{l, i}=\sum_{j} \frac{L_{j}}{2} \Omega_{j} U_{j}\left(T_{i}-T_{\infty, j}\right)
$$

Then the global heat transfer coefficient is calculated using expression (2.16), here $h_{j}$ stands for the convection heat transfer coefficient, calculated in expression (2.17), and $R_{R R, j}$ is the global heat transfer coefficient of the rock around the tunnel.

$$
U_{j}=\left(\frac{i}{h_{j}}+R_{R R, j}\right)^{-1}
$$




$$
h_{j}=\frac{1}{8} f_{j} c_{p, j} \frac{G_{j}}{A_{j}} .
$$

2.2.4. Mass transfer The mass transport in the $1 \mathrm{D}$ model is based on a combination of the continuity Equation and the Fick theory, according which the concentration in turbulent fluxes are proportional to the mean gradient concentration [4]. The one-dimensional form of this equation is in expression (2.18).

$$
\frac{\partial C}{\partial t}+u \frac{\partial C}{\partial x}=D \frac{\partial^{2} C}{\partial x^{2}}+S_{p}
$$

Here $C$ is the concentration of the different components, $D$ the diffusion tensor and $S_{p}$ is a source term for other contaminants. Integration of Eq. (2.18) in a control volume centered in one node gives expression (2.19)

$$
\int_{C V} \frac{d C}{d t} \partial V+\sum_{j} u_{j} A_{j} C_{j}=\sum_{j} D_{j} A_{j} \frac{C_{i}^{t}-C_{j}^{t}}{L_{j}}+S_{p, i}
$$

The concentration $C_{j}$ at each inlet section of the control volume is obtained using the upwind scheme, as in the thermal model. Following this scheme, the value of $C_{j}$ is the concentration of the upstream node considering the flow direction. The numerical expansion of the time dependent term involves the use of a backward Euler formulation, namely (2.20)

$$
\frac{C_{i}^{t}-C_{i}^{t-\Delta t}}{\Delta t}+\sum_{j} u_{j}^{t} A_{j} C_{j}^{t}=\sum_{j} D_{j} A_{j} \frac{C_{i}^{t}-C_{j}^{t}}{L_{j}}+S_{p, i}
$$

2.2.5. Matrix Formulation The equations previously written for a single control volume can be expressed for the entire network using a matrix formulation. In particular, the incidence matrix provides a mathematical description of the entire topology, linking the branches and the nodes. The continuity equation becomes Eq. (2.21).

$$
\vec{A} \Delta \boldsymbol{G}^{t}+\boldsymbol{G}_{E X T}^{t}+\boldsymbol{r}=0
$$

The vector $\boldsymbol{G}^{t}$ contains the flow rates in the branches at current time t. The vector $\boldsymbol{G}_{E X T}^{t}$ contains the flows extracted (if positive, injected if negative) at each node. The time dependent term is organized in the vector $\boldsymbol{r}$. If the assumption of incompressible flow is considered this term drops. The momentum equation becomes Eq. (2.22).

$$
\vec{A}^{T} \Delta \boldsymbol{P}^{t}=\left(\boldsymbol{R}^{t}+\boldsymbol{C}^{t}\right) \Delta \boldsymbol{G}^{t}-\boldsymbol{t}^{t}-\boldsymbol{s}^{t}
$$


The left-hand side of the equation represents the pressure variation in the branches, as $\mathbf{P}$ contains the total pressure in all the nodes. The term $R^{t} \Delta G^{t}$ accounts for the losses due to friction, being the matrix $R^{t}$ a diagonal matrix containing the fluid dynamic resistance of the various branches. The terms in $R^{t}$ depend on the mass flow rates, therefore an iterative calculation is necessary, as explained below. The vector $t^{t}$ is a generalized term containing the pressure variations due to the different sources (fans, vehicles), $s^{t}$ and $C^{t}$ are the contributions of the time dependent term, generally neglected.

With regards to the thermal model, the matrix form is in Eq. (2.23). This formula includes the diagonal mass matrix $M^{t}$, the stiffness matrix $K^{t}$ and the temperature vector $T^{t}$. On the right-hand side, the vector $f^{t}$ contains the known temperatures (as boundary conditions), $M^{t-\Delta t} T^{t-\Delta t}$ is the product of the mass term and temperatures in the nodes at the previous time step and $\Phi_{v, i}$ is a heat source term.

$$
\left(\boldsymbol{M}^{t}+\boldsymbol{K}^{t}\right) \boldsymbol{T}^{t}=\boldsymbol{f}^{t}+\Phi_{v, i}+\boldsymbol{M}^{t-\Delta t} \boldsymbol{T}^{t-\Delta t}
$$

The formulation for the mass transport model (2.24) has the same structure as the thermal model. The notation used is similar to the one used in (2.23). On the lefthand side, a mass term and a stiffness term multiplying the vector of concentrations. On the right-hand side are present the known terms, the source term of contaminants and the mass and concentration at the previous time step.

$$
\left(\boldsymbol{M}_{C}^{t}+\boldsymbol{K}_{C}^{t}\right) \boldsymbol{C}^{t}=\boldsymbol{f}_{C}^{t}+\boldsymbol{S}_{p}^{t}+\boldsymbol{M}_{C}^{t-\Delta t} \boldsymbol{C}^{t-\Delta t}
$$

2.2.6. Solution Procedure The method used to solve the fluid dynamic model is the SIMPLE algorithm (Semi-Implicit Method for Pressure Linked Equations), proposed by Patankar and Spalding [31]. This algorithm is based on a "guess and correct" procedure and uses a modified form of the matrix formulation, to solve the linkage between the equations in the fluid dynamic model.

The procedure the SIMPLE adopts is shown in Fig. 2, where superscripts * and 'refer to the guess values and to the corrections, respectively.

The term Var indicates the general dependent quantity. At the first iteration, both $\operatorname{Var}_{\text {old }}$ (the previous value) and Var* (the current approximation) assume the value at last time step. The 6 equations on the right are used to update the variables, starting with the mass flow. The first equation requires an iterative method to be solved, as the equations are non linear; the fixed point method can be applied to this purpose. The correction term to the pressures is then calculated using the second formula and is used with an under-relaxation factor to obtain the new pressure approximation (third equation). After this, the mass flow rate term is updated using an under-relaxation factor (fourth equation). Finally, the temperature and the concentration are updated (fifth and sixth equation). After obtaining the new values of the properties, the residuals of the expressions of the pressure and mass flow rates are used to evaluate the solution convergence. When 


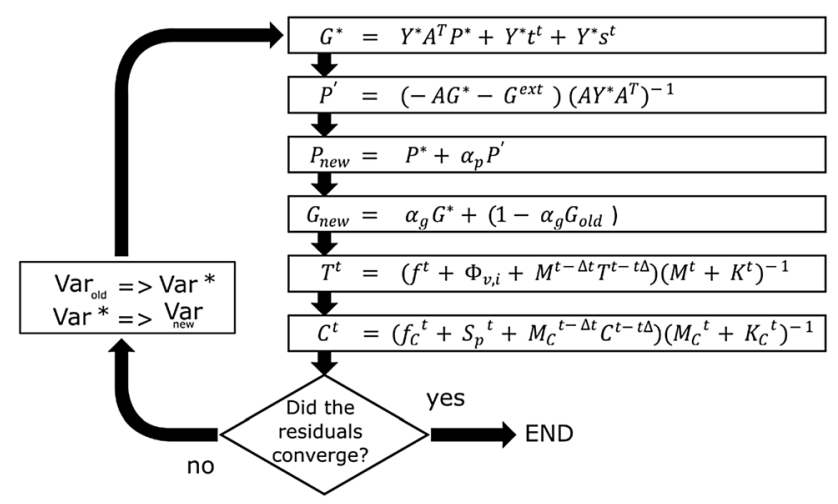

\section{Figure 2. SIMPLE Algorithm Procedure.}

the solution converges the SIMPLE exits, otherwise the values of the variables are saved and updated.

\subsection{D Descriptions and Characteristics}

The software used to run the 3D part of the algorithm is the FDS in its release version 6.7. FDS is an open source program, coded in Fortran 90 created to solve a wide variety of fire engineering problems and study the fundamentals of fire dynamics. Some main characteristics can be pointed out of the FDS:

- Geometry It uses meshes and obstructions built using quadrilateral shapes. Multiple meshes can be simulated in parallel, to optimize the computational time.

- Hydrodynamic Model The turbulent model used is the LES (Large Eddy Simulation). The solution is calculated using a two-step "predictor corrector" algorithm to solve the Navier-Stokes equations.

- Combustion model The default combustion model uses a single step, mixing-controlled reaction (meaning mixed is equal to burnt). This reaction uses only 3 reactants: air, fuel and products, lumping them for easier handling.

For more details on how the FDS works we refer the reader to the different guides included with the FDS, the User Guide and the Technical Reference Guide $[19,32]$.

\subsection{Interaction Between the $1 D$ and $3 D$ Models}

The interaction between the two models can be classified, according to previous works in the mathematical theory for domain decomposition [33], as a non-overlapping Dirichlet-Neumann Direct Coupling. Its main characteristics are: (1) meshes of the 1D and 3D do not share any common domain. (2) Dirichlet and Neumann boundary conditions are set on each common boundary. (3) a constant exchange of information occurs between the two modules during the simulation, where both sub-algorithms are ran together. 
To manage the coupling between the two models, proper modifications have been implemented on both FDS and Whitesmoke. As a first step, the models have been joined through their codes, compiling them together. To achieve this, subroutines have been created to manage the data exchange among the 3D and the 1D. A new namelist has been introduced to FDS, called $\mathrm{EXCH}$, to introduce the data regarding the exchange boundaries between the two models. The information exchange and 1D calculation are done at the end of the FDS timestep, leaving the boundary conditions for the next timestep.

The boundary conditions that can be used to link FDS with Whitesmoke are two: the "PRES" and the "VEL" boundaries.

2.4.1. "PRES" Boundary In the "PRES" boundary condition, the 3D model imposes the pressure to the 1D model and the 1D model imposes the mass flow rate to the 3D model. This boundary behaves as a vent in FDS, meaning that it only has a mass flow in one direction, entering or exiting the domain. The pressure value that the $3 \mathrm{D}$ imposes is calculated as the average value of the pressure, weighted by the mass flow per unit area, in the boundary section and is assigned as the pressure of the 1D node in the boundary, not the total pressure. The mass flow of the 1D is imposed as the mass flow of the surface in the exchange boundary (in the $3 \mathrm{D}$ ).

Therefore, mass is conserved using the mentioned approach, where the mass flow is calculated in the 1D and then evenly assigned to the 3D boundary. And the momentum is also conserved by exchanging the pressure, calculating the average of the pressure times the mass flow, introducing the mass flow averaged pressure as the $1 \mathrm{D}$ boundary condition.

2.4.2. "VEL" Boundary In the "VEL" boundary, the 3D model imposes the mass flow rate to the $1 \mathrm{D}$ model, and the $1 \mathrm{D}$ model imposes the pressure to the $3 \mathrm{D}$ model. In FDS, this boundary condition behaves as an open boundary. Open boundaries allow flow in both directions at the same time; therefore, backflow is allowed. Even as this surface does not present issues in terms of backflow, the flow must be as homogeneous as possible to ensure a good transition from the $3 \mathrm{D}$ to the 1D part of the algorithm.

In this boundary, the mass conservation is ensured as the mass flow, per unit area, is calculated in the $3 \mathrm{D}$, as the average of the product of the density and velocity in each cell of the boundary; and then assigned to the 1D node multiplied by the area. And the pressure of the 1D node is introduced as a dynamic pressure (an FDS parameter for OPEN surfaces), in the 3D boundary.

2.4.3. Boundary Considerations Tunnel simulations often use as boundary conditions velocities, as measured in a tunnel section or ventilation shafts, and environmental pressure exits. This are the boundary conditions considered to design the multiscale model. Therefore, both the "PRES" and "VEL" boundaries are used at least once, in the cases shown. 
Simulations with two "PRES" boundaries, imposing the velocity at both sides of the tunnel have not been considered to avoid mass accumulation in the $3 \mathrm{D}$ domain, caused by having fixed mass flows at both ends.

Then, the position of each boundary should be decided. In our simulations, the "PRES" boundary condition is always placed upstream of the fire, to keep it away from backflows, that cannot be handled. It is placed at a distance higher than the backlayering length, being this quantity calculated using one of the different expressions available in the literature [16, 34, 35]. In cases where there is no backlayering or it is considerably short, still some distance must be left for the flow to pass from laminar to turbulent; the distance will depend on the flow speed. Opposingly, the "VEL" boundary is located downstream the fire, at a distance long enough to get a homogeneous flow when the simulation reaches a steady state. A schematic example can be seen in Fig. 3.

2.4.4. Temperature and Concentration Boundary Conditions The temperature and concentration boundaries are calculated in a way that is independent of the type of boundary.

Temperature and concentration are imposed along the mass flow direction, therefore, if the flow goes from the $3 \mathrm{D}$ to the $1 \mathrm{D}$, the $3 \mathrm{D}$ will impose the air concentration, and temperature, in that boundary, and vice versa. These quantities are calculated in the 3D boundary, averaging with respect to the mass flow rate and assigning it to the 1D node; and from the 1D assigning the 1D node temperature, or concentration, to the 3D boundary.

2.4.5. Multiscale Accuracy and Stability The accuracy and stability of the model must be separated in three parts, $1 \mathrm{D}, 3 \mathrm{D}$ and the interface.

The 3D, accuracy and stability of the FDS as a CFD model has been widely tested, in both scientific articles and their own guide [36, 37]. The FDS boundary conditions inside the multiscale change at each timestep of the 1D model (e.g. every $3 \mathrm{~s}$ of simulation time). This to benefit stability, as changes in the boundaries introduce changes in the whole tunnel that require a short number of timesteps to be assimilated by the model.

The $1 \mathrm{D}$, accuracy and stability of the Whitesmoke are related to the solver and, mostly, the tolerances used. The SIMPLE [31] solver is used. This is a "guess and correct" algorithm. To ensure convergence and validity of the results, four criteria are used, three of them signal that the error between the last two iterations in any point must be lower than $10^{-2}$ for temperature, concentration and mass flow, and the last one checks the residuals of the momentum equation, which should also be

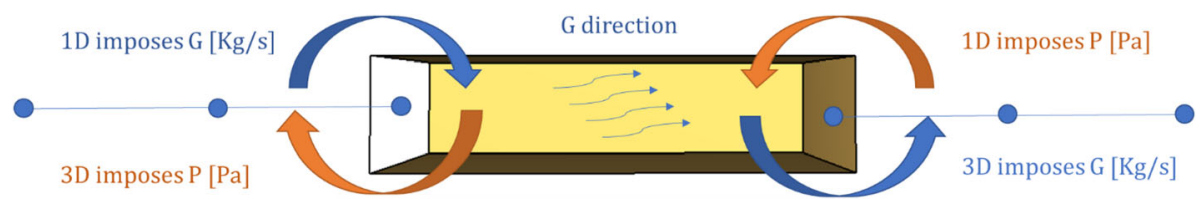

Figure 3. Boundary Placement. 
lower than $10^{-2}$. To achieve faster convergence the solver uses under-relaxation factors to reduce the oscillations of the solution.

The Interface, the way the information is exchanged has already been explained in this section. The exchange is done at the end of the FDS iteration to not interfere in the FDS solver process. Then the boundary conditions of FDS are changed with different 1D data, depending on the kind of boundary, to be used in the next FDS iteration. The data passed from one domain to the other is averaged in the mass flow, and then distributed accordingly in the other domain boundary, to reduce possible instabilities.

\subsection{Interaction with the Ventilation System of a Tunnel}

The model must consider both the tunnel and the ventilation system. Depending on the configuration, ventilation can be classified as longitudinal, transversal or semi-transversal. The three configurations are shown in Fig. 4; these can be built and effectively calculated using the 1D-3D model. Using the novel features of the model that are presented here, it is possible to: (1) precisely model the jet-fans and axial fans in the 1D portion, introducing proper characteristic equations, (2) consider the operational degradation of these devices due to smoke propagation, (3) consider the stack effect and the piston effect in the 1D portion, (4) impose the pressures at the portals.

The components needed to build these kinds of configurations are fans, ducts and extractions, and can be introduced in various ways, as discussed below.

2.5.1. Extractions and Networks Extractions are outlets for the ventilation systems of tunnels, mostly for long tunnels. It is possible to add extractions to the multiscale model in its $1 \mathrm{D}$ or $3 \mathrm{D}$ part as shown in Fig. 5. For the $3 \mathrm{D}$ part FDS vents

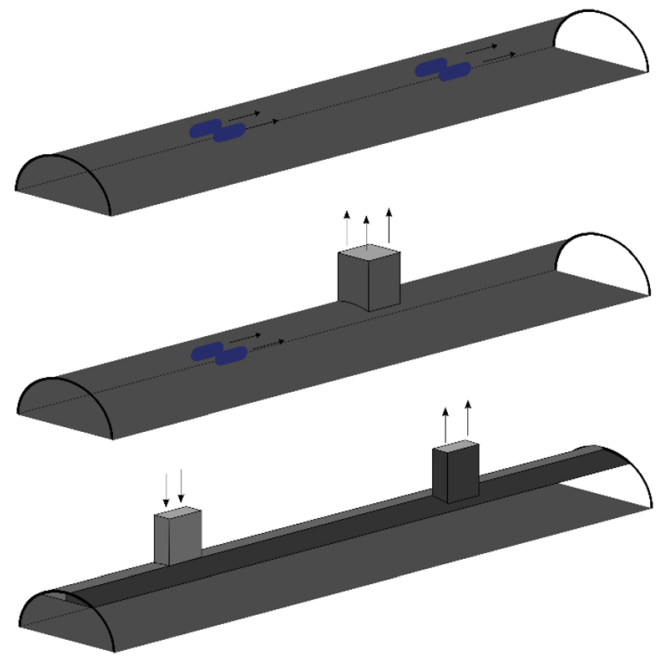

Figure 4. Different types of ventilation, Longitudinal, semitransversal and transversal. 


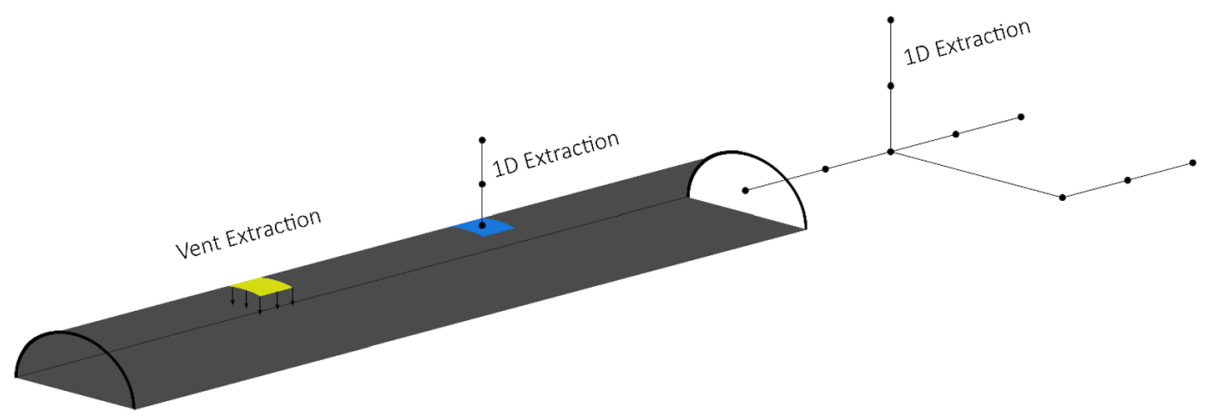

\section{Figure 5. Extraction configurations.}

can be used, fixing in this way the volumetric or mass flow of the extraction. Otherwise, a boundary to the $1 \mathrm{D}$ can be used to simulate the extraction. In this way, the boundary connects to a node that ultimately arrives to a boundary with a mass flow or pressure condition.

Tunnel networks can also be simulated with the $1 \mathrm{D}$, different bifurcations and complex tunnels can be introduced, the only requirement is to discretize them into nodes and branches and introduce them into the 1D.

2.5.2. Fans Jet fans are mostly used in longitudinal and semi-transversal ventilation systems to induce a longitudinal velocity in the air flow. From a modelling perspective, fans also give a flow boundary condition, which causes a pressure rise in the section where they are installed. They can be modelled as shown in Fig. 6 . In the 3D part of the model, fans can be introduced through regular FDS methods, for example, a couple of vents in an obstruction introducing and extracting air. When multi-scale is adopted fans can be easily added to the 1D part of the model, specifying the position where they are installed and their characteristic curve or some operational properties of the fan. More Details regarding the modelling of the fans are in [3].

2.5.3. Ducts At last, ducts are mainly used in semi-transverse ventilation systems. Ducts are used to manage the air flow and communicate to ventilation shafts.

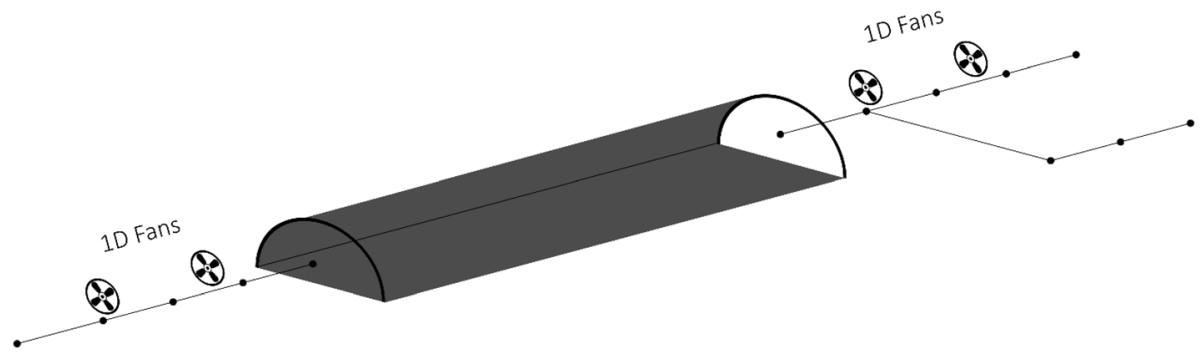

Figure 6. Fans configurations. 
Ducts can be introduced by placing 1D boundaries in the tunnel and building the corresponding 1D network. In this case, the advantage of using Whitesmoke is that it is possible to consider the characteristic curve of the fan and then calculate the air/exhaust flow as the function of the external and internal pressures and considering the temperature distribution.

2.5.4. Local Effects Another important advantage of using multiscale is that it is much easier to take local effects into account. These include for instance the presence of obstacles to the air/exhaust flow but also the piston effect. The latter is typically a transient effect due to the vehicles moving in the tunnel, therefore it is quite difficult to consider it in the 3D model and much easier in the $1 \mathrm{D}$ model.

\subsection{Multiscale Model Application}

The proposed 1D-3D represents the networks in the same way as the FDS HVAC, treating the data differently. Both models calculate quantities as the losses (both minor and flow losses), the mass fractions, among others. But the 1D-3D adds the capabilities to calculate the heat losses through the network, impose other kinds of pressure boundaries, as pressurized and velocity boundaries, and add inclination or special pressure changes to the $1 \mathrm{D}$ calculation. The $1 \mathrm{D}-3 \mathrm{D}$ features a direct coupling between both algorithms, while FDS and HVAC has a non-direct coupling and may struggle with big inlets or outlets, as it was not meant for them.

2.6.1. Test Case To test the capabilities of the proposed approach, a tunnel is simulated with FDS only and the multiscale model. The simulated tunnel is a onetrack tunnel with dimensions of $4.8 \mathrm{~m} \times 4.8 \mathrm{~m} \times 600 \mathrm{~m}$. The walls have a roughness of $4.2 \mathrm{~mm}$ and are modelled as inert walls at a fixed temperature (as the difference with simulating the heat exchange through the wall thickness resulted in similar temperatures and heat losses).

The Heat source is placed at a distance of $0.3 \mathrm{~m}$ from the floor in the midpoint of the section and tunnel length. The fire comes from a pool of Diesel, with a Heat of combustion of $43,027 \mathrm{~kJ} / \mathrm{kg}$ and a soot yield of $5 \%$. The pool dimensions are $1.2 \mathrm{~m} \times 1.2 \mathrm{~m} \times 0.3 \mathrm{~m}$. The heat release rate of the fire is of $2 \mathrm{MW}$, as in Fig. 7. This fire intensity was selected considering future validations of the model with an experimental test and that they would require heat rates that guarantee

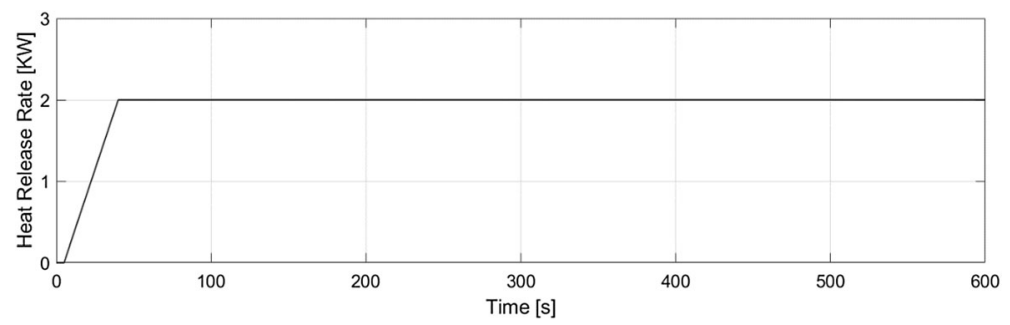

Figure 7. Heat Release Rate of the Fire Source. 
the integrity of the test site or tunnel. The air velocity is imposed equal to $1 \mathrm{~m} / \mathrm{s}$ on the upstream portal and an external temperature of $15^{\circ} \mathrm{C}$ is considered.

The comparison is performed between a full FDS simulation $(600 \mathrm{~m})$ and a multiscale simulation (a 1D section of $150 \mathrm{~m}$, a 3D section of $250 \mathrm{~m}$ and a second 1D section of $200 \mathrm{~m}$ ), as shown in Fig. 8. The 1D has calculating nodes at the boundaries, at $200 \mathrm{~m}$ and $300 \mathrm{~m}$ of distance to the fire, both up and downstream.

The distances from the fire to the boundaries were selected based on different criteria. First, the upstream distance is constrained by the backlayering length, the backlayering expected for the characteristics of this fire was around the $80 \mathrm{~m}$ (calculated using the relationships found in [16]), so $150 \mathrm{~m}$ were simulated in $3 \mathrm{D}$ to ensure that the smoke would not arrive to the boundary.

In the downstream position, it is necessary to guarantee that the flow arrives to a homogeneous or quasi-homogeneous state. The ideal length would let the flow arrive to environmental temperature but depending in the heat release rate of the fire, and other factors in smaller degree, it would reduce the time saving of the whole simulation. Therefore $100 \mathrm{~m}$. were set in the downstream site as it represented a distance were the flow properties descended with a monotone steepness towards environmental conditions.

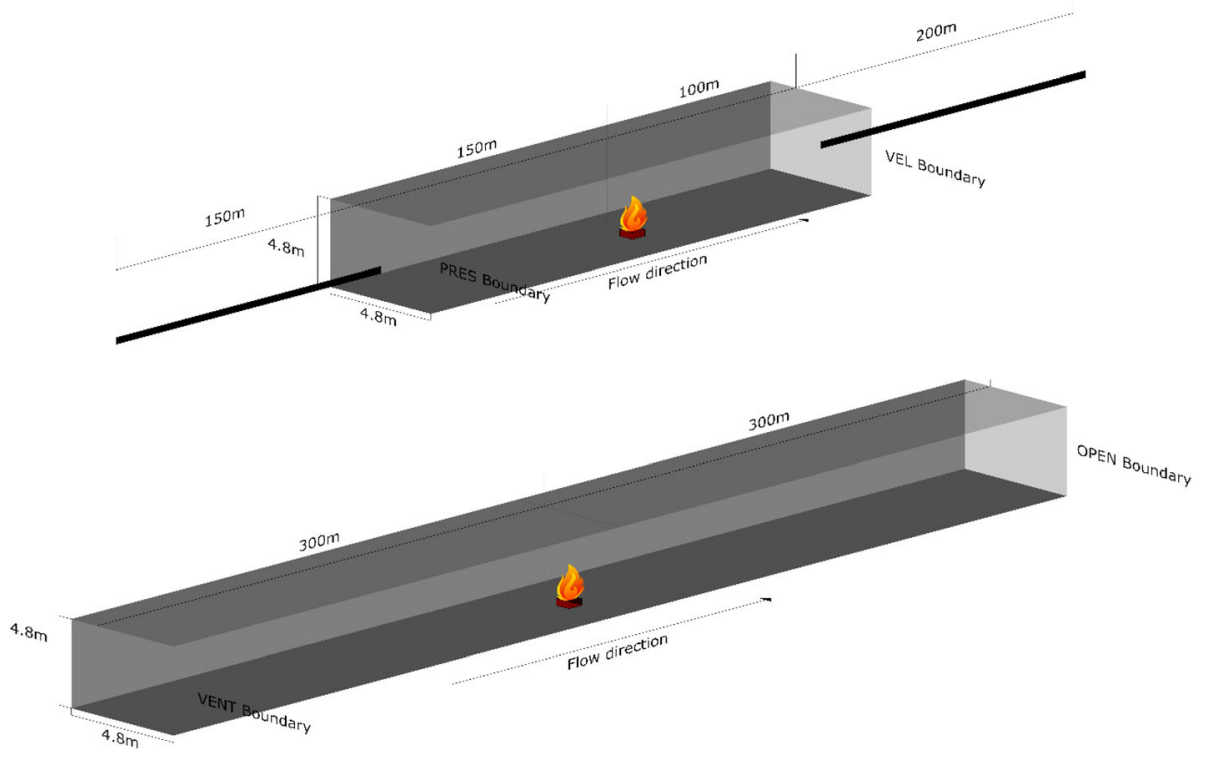

Figure 8. Simulations in the Multiscale (upper), and FDS (lower). 
(a)

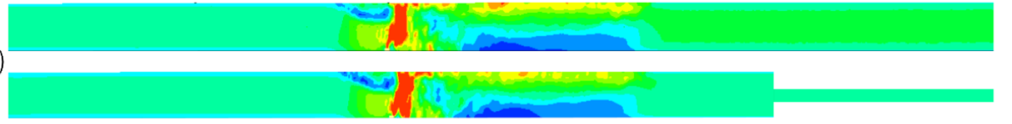

(b)

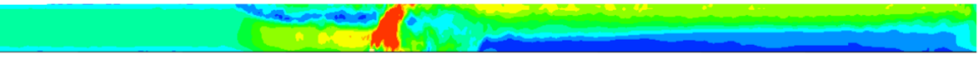

(b)

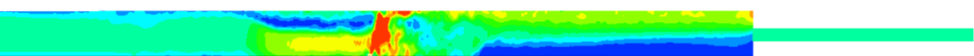

c)
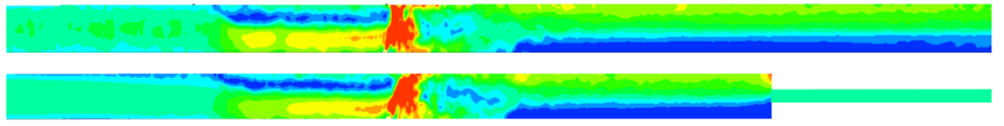

(d)
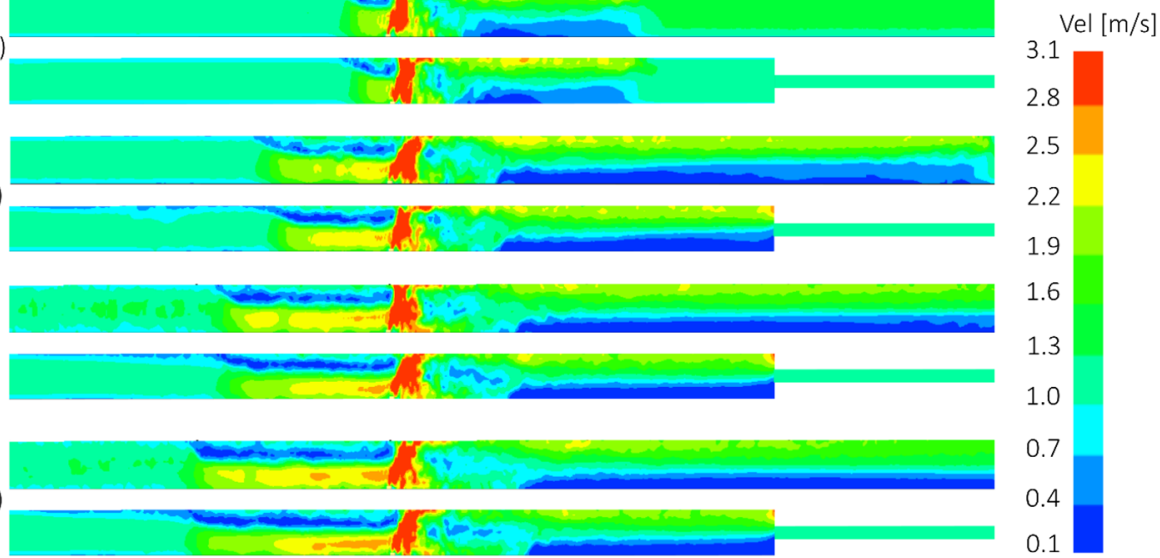

\section{Figure 9. Velocity $[\mathrm{m} / \mathrm{s}]$ distribution at the boundary at a $50 \mathrm{~s}, \mathrm{~b}$} $100 \mathrm{~s}, \mathrm{c} 150 \mathrm{~s}$ and $\mathrm{d} 200 \mathrm{~s}$.

\section{Results}

The output from FDS and the Multiscale model is shown next. In Fig. 9, the distribution of velocities can be appreciated along the tunnel, for the multiscale and full $3 \mathrm{D}$ cases. Here the $1 \mathrm{D}$ region is drawn slimmer to distinguish it from the $3 \mathrm{D}$ domain, still it has the same cross-section in the calculations. It can be noticed that the backlayering stops at close to $80 \mathrm{~m}$, for the upstream part of the tunnel, with almost the same backlayering length for both simulations. Also, the velocity in the downstream part of the tunnel is the mean value in the section, which is representative of the whole cross section. This result shows that the velocity profiles are similar in the two cases, with a deviation smaller than $6 \%$ on the backlayering length.
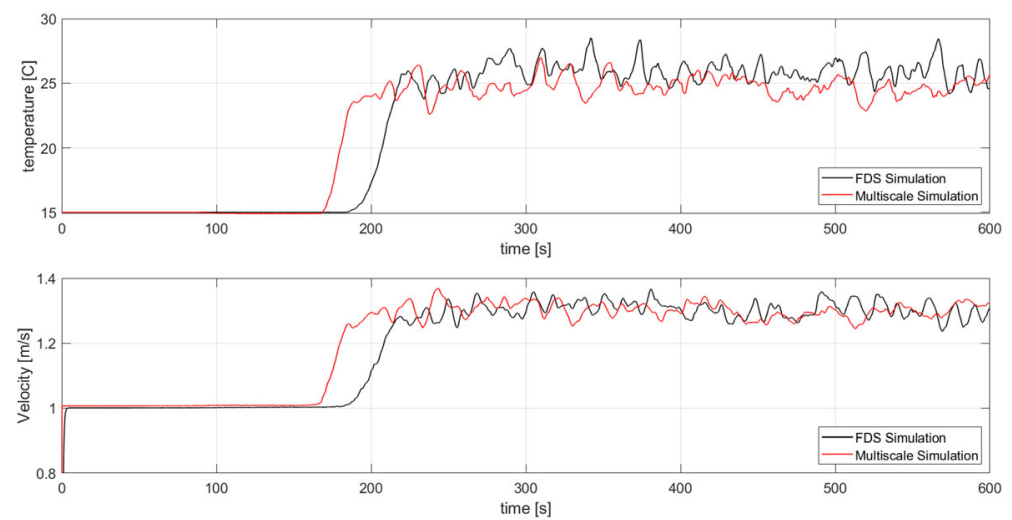

Figure 10. Velocity and Temperature $50 \mathrm{~m}$ upstream of the fire, mass averaged value. 
Figures 10 and 11 compare the temperature and velocity in two sections of the tunnel, $50 \mathrm{~m}$ upstream in Fig. 10 and $50 \mathrm{~m}$ downstream in Fig. 11 of the fire. In both comparisons the error introduced by the multiscale model is negligible in the steady state value. In Fig. 10 is possible to observe that the values are offset for some seconds, but the error disappears in a short amount of time.

Figures 12, 13 and 14 show the comparison of the longitudinal evolution of average temperature and average velocity (both as a magnitude and in the tunnel axis) obtained at the steady state using the two models. Three sections can be analysed in the two graphs.

First, the upstream section where the velocities and temperatures match closely from one simulation to the other. In this part, our model achieves great time savings without adding error to the whole simulation. Therefore, as long as the backlayering length of the fire is respected the $1 \mathrm{D}$ can be used without consequences. A difference of less than $5 \mathrm{~m}$ is observed in the backlayering length, observable in both the pressure and temperature graphs.

The second part would be the fire zone. In the vicinities of the fire is appreciated how both simulations match almost completely. This indicates that the assumptions and boundaries imposed by the multiscale model do not affect the results obtained from the fire zone in the FDS calculation. Therefore, evaluating the fire and its effects in the vicinities has the same results in the FDS by itself or being paired in the multiscale model. The maximum difference in the average temperature is in the cross section is about $4{ }^{\circ} \mathrm{C}$, while the difference in the average velocity is less than $0.05 \mathrm{~m} / \mathrm{s}$.

The zone after the fire gets similar behaviours with temperatures with the tendency to decrease, as heat is loss through the walls, and velocities that also decrease, as the air gets denser. Still in this last part there are two zones where the results are moderately different. These zones match the places where the boundaries of the domains are placed. Both boundaries are OPEN surfaces from the FDS and are characterized by a release of the flow to an environmental condition.
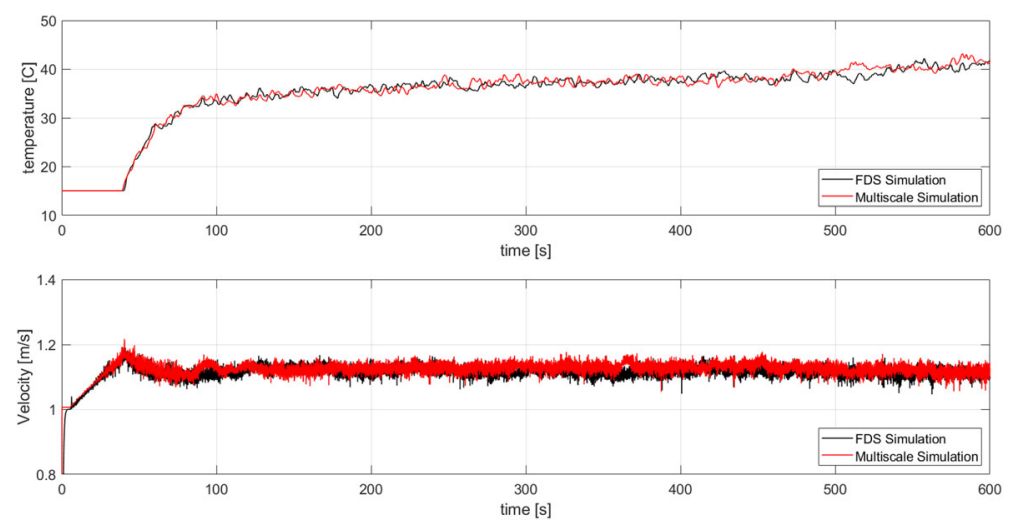

Figure 11. Velocity and Temperature $50 \mathrm{~m}$ downstream of the fire, mass averaged value. 


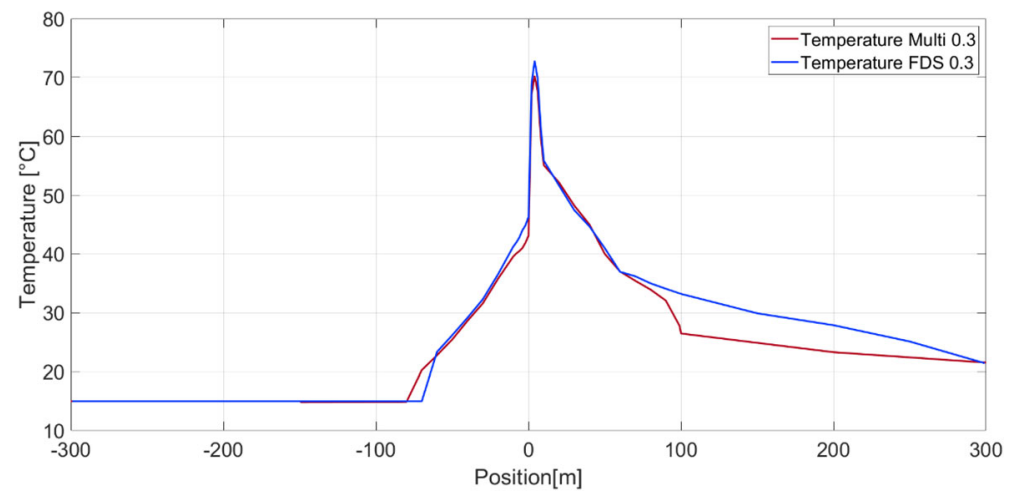

Figure 12. Comparison of Temperature between a full 3D simulation and a Multiscale simulation.

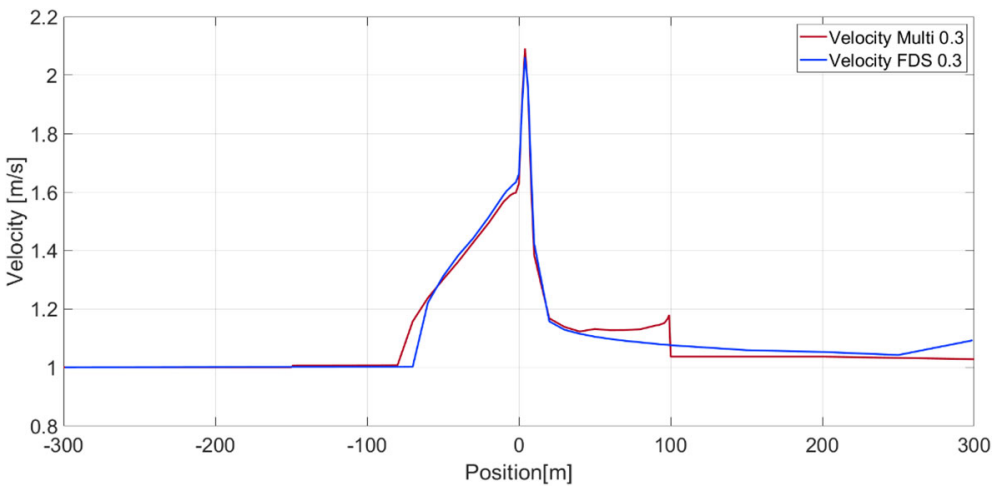

Figure 13. Comparison of velocity (magnifude) between a full 3D simulation and a Multiscale simulation.

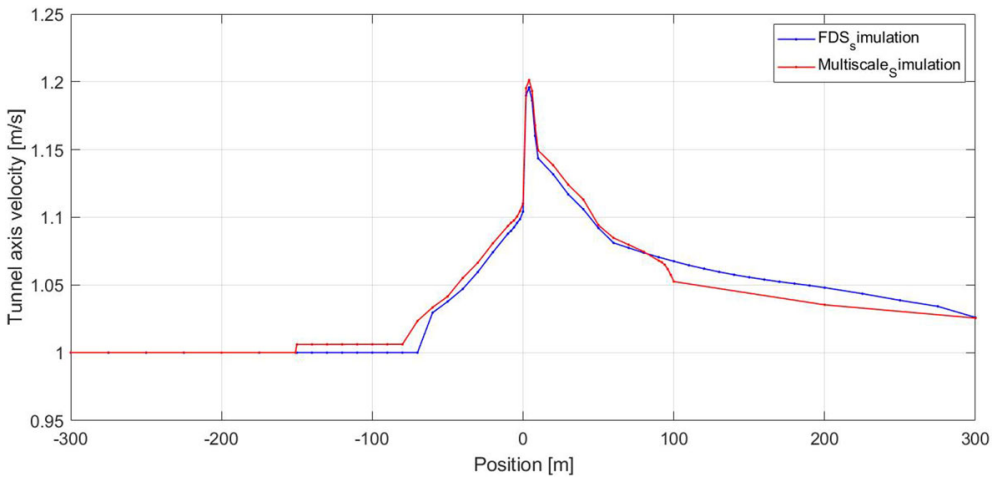

Figure 14. Comparison of velocity (tunnel axis component) between a full 3D simulation and a Multiscale simulation. 
This flow release involves a flow velocity increase in the less dense part of the section, and a recirculation from environmental air in the denser region, as observed in the Fig. 10.

The behaviour obtained in Fig. 15 is a consequence of buoyancy. The less dense upper region obtains a vertical velocity increase due to the density difference with environmental air of the outside. And as a consequence of the increase of velocity and mass flow in the upper part, the lower part presents recirculation to conserve the mass balance. The consequence of this interaction is a drop in the temperature, as colder air comes into the domain, and an increase in the velocity, due to buoyancy. In Figs. 12, 13 and 14 this effect is observed close to the 100th meter downstream the fire, in the multiscale simulation, introducing an error in temperature and velocity. The effect appears again in the $300^{\text {th }}$ meter downstream, but now in the FDS calculation. This behaviour is correct when the outlet section coincides with a tunnel portal, while it is not in the case of a 3D-1D link. Depending on the average temperature of the exhausts on this section, the effect might require corrections. A possible approach consists in partially superimposing the $3 \mathrm{D}$ and the $1 \mathrm{D}$ sections, so that the temperatures and velocities in the portion of tunnel affected by this issue are calculated using the 1D model.

Regardless of the issues in the boundary, in Fig. 16 is possible to observe that the mass flow that enters the tunnel is conserved in its way to the exit, with some oscillations because of the unsteady nature of fire simulations. Furthermore, in the first half of the simulation we can observe that the mass flow value is greater, this matches with the heating and expansion of the air inside the tunnel. This effect disappears progressively as the tunnel reaches a steady temperature in all its length, around $400 \mathrm{~s}$.

At last, Fig. 17 shows that the time reduction obtained using the Multiscale model, compared to the full-scale model. The reduction amount is proportional to the region of the simulation that is calculated using the 1D model of the multiscale. In this case the 1D calculates about $58 \%$ of the length and a reduction close to the $50 \%$ of the time is obtained. It must be remarked that this calculation time reduction should increase for longer tunnels with lengthier parts being processed by the 1D model. Therefore, the benefits of the model should be bigger when used in the longer tunnels, for which it is though.
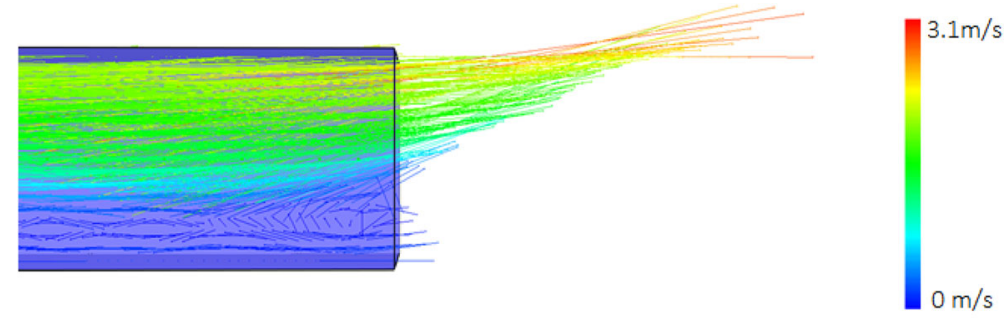

Velocity $[\mathrm{m} / \mathrm{s}]$

Figure 15. OPEN boundary behavior. 


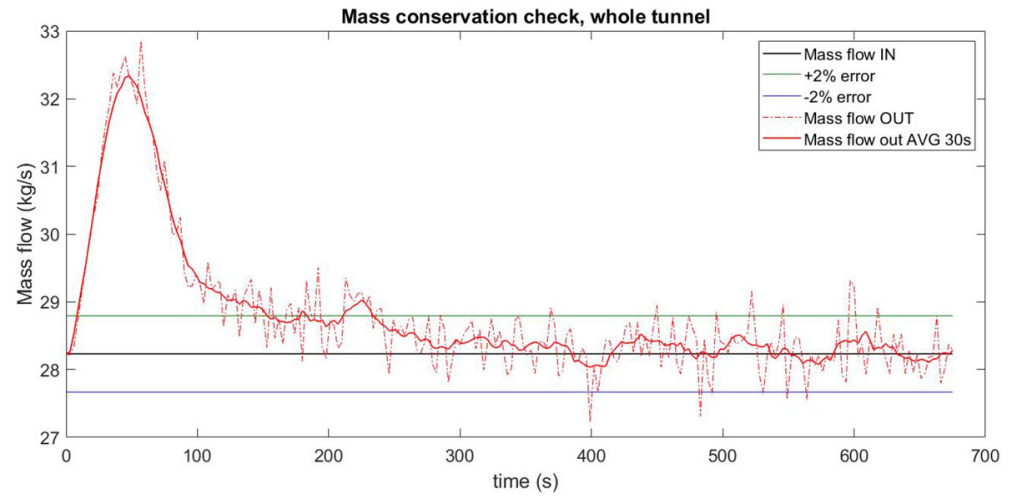

Figure 16. Mass conservation, comparisom of the flow entering and leaving the domain.

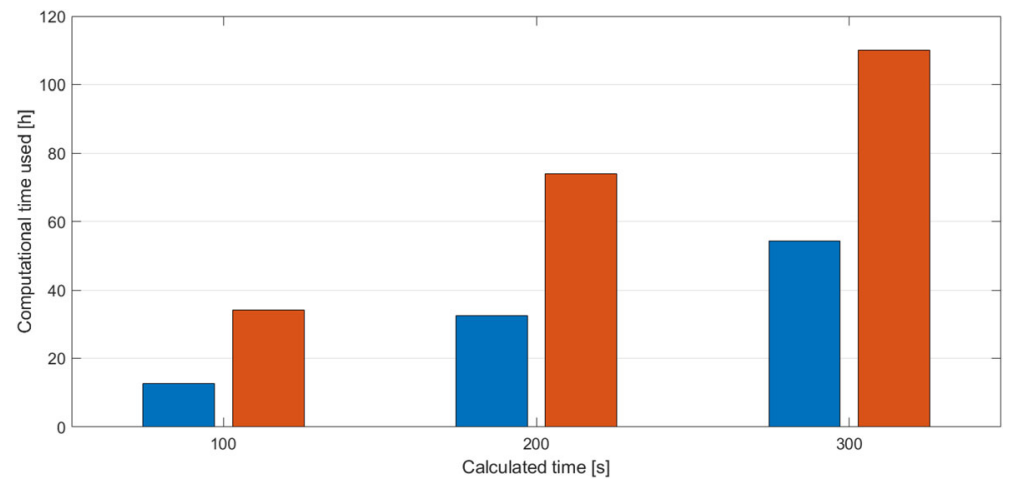

Figure 17. Amount of calculation time required using a $0.25 \mathrm{~m}$ mesh for different amounts of simulation time.

\section{Conclusions}

This paper proposes a multiscale 1D-3D model for application to tunnels in the case of fire. The model is obtained by joining together the CFD code FDS and the 1D code Whitesmoke. The direct coupling between the two models provides a constant exchange of information among them, giving the whole code robustness. With respect to the full $3 \mathrm{D}$ simulation, the model is capable of reducing the computational time proportionally to the $1 \mathrm{D}$ portion of domain. In addition, the simulation capabilities are enhanced as the approach allows one considering pressure boundary conditions at the tunnel portals or at the ventilation shafts (which is not straightforward with full 3D simulation). Moreover, the proposed approach is expected to allow one incorporating the features of the code Whitesmoke: (1) the characteristic curves of the fans/jet-fans and the degradation effects due to smoke propagation can be considered, (2) the piston effect can be properly considered. 
A comparison between the two models shows that results are very close, provided that the backlayering distance is respected upstream and that enough distance is modelled in the $3 \mathrm{D}$ downstream. The distance required downstream is case sensitive and depends mainly in the flow speed and the fire heat release rate. The error the multiscale introduces into the simulation is localized in the OPEN boundaries it uses. A future development for the model would be focused towards the elimination of this irregularity by creating inside the code a Neumann boundary in temperature and velocity.

\section{Funding}

Open access funding provided by Politecnico di Torino within the CRUI-CARE Agreement.

\section{Open Access}

This article is licensed under a Creative Commons Attribution 4.0 International License, which permits use, sharing, adaptation, distribution and reproduction in any medium or format, as long as you give appropriate credit to the original author(s) and the source, provide a link to the Creative Commons licence, and indicate if changes were made. The images or other third party material in this article are included in the article's Creative Commons licence, unless indicated otherwise in a credit line to the material. If material is not included in the article's Creative Commons licence and your intended use is not permitted by statutory regulation or exceeds the permitted use, you will need to obtain permission directly from the copyright holder. To view a copy of this licence, visit http://creat ivecommons.org/licenses/by/4.0/.

\section{References}

1. Cheng LH, Ueng TH, Liu CW (2001) Simulation of Ventilation and fire in the underground facilities. Fire Saf J 36(6):597-619

2. Riess I, Bettelini M, Brandt R (2000) SPRINT a desing toll for fire ventilation. In: Proceedings of aerodynamics and ventilation of vehicle tunnels

3. Colella F (2010) Multiscale modeling of tunnel ventilation flows and fires, Ph.D. Thesis, Torino: Politecnico di Torino

4. Cosentino S (2016) Innovative modelling approaches for the design, operation and control of complex energy systems with applications to underground infrastructures. Ph.D. Thesis, Torino: Politecnico di Torino

5. Cosentino S, Mejias J, Borchiellini R, Verda V, Lupinacci F (2017) Integrating 1D and $3 \mathrm{D}$ modelling for the analysis of ventilation control in tunnels. In: International symposium on advances in computational heat transfer

6. Wade C, Barnett J (1996) A room-corner fire model including fire growth on linings and enclosure smoke-filling. J Fire Prot Eng 8(4):183-193 
7. Floyd J, Hunt S, Williams F, Tatem P (2005) A Network Fire Model for the simulation of fire growth and smoke spread in multiple compartments with complex ventilation. J Fire Prot Eng 15(3):199-299

8. Peacock R, Forney G, Reneke P, McGrattan K (2019) CFAST consolidate fire and smoke transport, technical reference guide, gaithersburg. National Institute of Standards and Technology, Maryland

9. Jones W, Forney G (1990) A programmer's reference manual for CFAST, the useful model of fire growth and smoke transport., Gaithersburg, Maryland: National Institute of Standards and Technology

10. Trouvé A, Wang Y (2010) Large eddy simulation of compartment fires. Int J Comput Fluid Dyn 24(10):449-466

11. Wahlqvist J, Hees P (2013) Validation of FDS for large-scale well-confined mechanically ventilated fire scenarios with emphasis on predicting ventilation system behavior. Fire Saf J 62:102-114

12. Jenft A, Collin P, Boulet G, Pianet A, Muller A (2013) Experimental and numerical study pool fire suppression using water mist. Fire Saf J 67:1-12

13. Chow WK, Gao Y, Zhao JH, Dang JF, Chow N (2016) A study on tilted tunne fire under natural ventilation. Fire Saf J 81(Supplement C):44-57

14. Li YZ, Fan CG, Ingason H, Lonnermark A, Ji J (2016) Effect of cross section and ventilation on heat release rates in tunnel fires. Tunn Undergr Space Technol 51(Supplement C):414-423

15. Li Y, Ingason H (2017) Effect of cross section on critical velocity in longitudinally ventilated tunnel fires. Fire Saf J 91(Supplement C):303-311

16. Hsu W, Huang Y, Shen T, Cheng C, Cheng T (2017) Analysis of the Hsuehshan tunnel fire in Taiwan. Tunn Undergr Space Technol 69(Supplement C):108-115

17. Liles D, Mahaffy J (1986) TRAC-PF1/MOD1: An advanced best-estimate computer program for pressurized water reactor thermal-hydraulic analysis. Los Alamos National Lab, Los Alamos

18. George T (2006) GOTHIC containment analysis package users manual. Numerical Applications Inc., Richland

19. McGrattan K, Hostikka S, McDermot R, Floyd J, Vanella M (2020) Fire dynamics technical reference guide, Volume 3: Validation, Gaithersburg, Maryland: National Institute of Standards and Technology

20. Passerini T, de Luca M, Formaggia L, Quarteroni A,Veneziani A (2009)A 3D/1D geometrical multiscale model of cerebral vasculature. J Eng Math 64(319)

21. Colella F, Rein G, Borchiellini R, Torero J (2011) A novel multiscale methodology for simulating tunnel ventilation flows during fires. Fire Technol 47(1):221-253

22. Colella F, Rein G, Verda V, Borchiellini R (2011) Multiscale modelling of transient flows from fire and ventilation in long tunnels. Comput Fluids 51(1):16-29

23. Colella F, Rein G, Verda V, Borchiellini R, Torero J (2011) Time-dependent multiscale simulations of fire emergencies in longitudinally ventilated tunnels. Fire Saf Sci 10:359-372

24. Haghighat A, Luxbacher K, Lattimer B (2018) Development of a Methodology for interface boundary slection in the multiscale road tunnel fire simulations. Fire Technol, 54(4)

25. Ang C, Rein G, Peiro J, Harrison R (2016) Simulating longitudinal ventilation flows in long tunnels: comparison of full CFD and multi-scale modelling approaches in FDS6. Tunn Undergr Space Technol 52(Supplement C):119-126

26. Vermesi I, Rein G, Colella F, Valkvist M, Jomaas G (2017) Reducing the computational requirements for simulating tunnel fires by combining multiscale modelling and multiple processor calculation. Tunn Undergr Space Technol 64:146-153 
27. Chandrashekar M, Wong F (1982) Thermodynamic system analysis- a graph-theoretic approach. Energy 7(6):539-566

28. ASHRAE (2013) ASHRAE Handbook-Fundamentals, Atlanta: the american society of heating, refrigerating and air-conditioning engineers

29. PIARC (1996) Road tunnels: emissions, environment, ventilation, PIARC

30. Sciacovelli A, Verda V, Borchiellini R (2013) Numerical design of thermal systems. CLUT, Torino

31. Patankar S (1980) Numerical heat transfer and fluid flow. Tayor and Franci, New York

32. McGrattan K, Hostikka S, McDermott R, Floyd J, Vanella M (2020) Fire dynamics simulator, user guide, Gaithersburg. National Institute of Standards and Technology, Maryland

33. Quarteroni A, Valli A (1999) Domain decomposition methods for partial differential equations. Oxford Science Publications, Oxford

34. Wu Y, Baker M (2000) Control of Smoke flow in tunnel fires using longitudinal ventilation systems- a study of the critical velocity. Fire Saf J 35:363-390

35. Li Y, Lei B, Ingason H (2010) Study of critical velocity and backlayering lenght in longitudinally ventilated tunnels fires. Fire Saf J 45:361-370

36. McGrattan K, Hostikka S, McDermott R, Floyd J, Vanella M (2020) FDS verification guide, sixth edition, Gaithersburg. National Institute of Standards and Technology, Maryland

37. McGrattan K, Hostikka S, McDermot R, Floyd J, Vanella M (2020) Fire dynamics simulator, validation guide, Gaithersburg. National Institute of Science and Technology, Maryland 